\title{
Intelligent Lane Reservation System for Highway(s)
}

\author{
Ciprian Dobre \\ Computer Science Department, University Politehnica of Bucharest, 060042 Bucharest, Romania \\ Correspondence should be addressed to Ciprian Dobre, ciprian.dobre@cs.pub.ro
}

Received 12 February 2012; Accepted 27 October 2012

Academic Editor: Yue Liu

Copyright (C) 2012 Ciprian Dobre. This is an open access article distributed under the Creative Commons Attribution License, which permits unrestricted use, distribution, and reproduction in any medium, provided the original work is properly cited.

\begin{abstract}
Highways tend to get congested because of the increase in the number of cars travelling on them. There are two solutions to this. The first one, which is also expensive, consists in building new highways to support the traffic. A much cheaper alternative consists in the introduction of advanced intelligent traffic control systems to manage traffic and increase the efficiency of the already existing highways. Intelligent lane reservation system for highways (ILRSH) is such a software control system. It is designed to assist and automate the use of a highway lane as a reserved lane. The idea is to allow and support drivers to travel at a speed higher, if in return they are willing to pay a small fee to reserve an empty virtual slot on the reserved lane. This slot is valid for a portion and of the highway and a time window, so each driver pays the fee depending thier its travelling needs. In return, drivers are guaranteed a congestion-free travel on that portion. In this paper, we present the proposed architecture of the ILRSH and its subsystems. The system is based on several proposed algorithms designed to assist the drivers, enter or exit the reserved lane, based on real-world driving observations. We present extensive simulation results showing the feasibility of the proposed approach, that can easily be implemented with little costs on already-existing highways, and the increase in traffic efficiency.
\end{abstract}

\section{Introduction}

Increasing passenger and freight travel led to growing congestions, with negative consequences on wasted energy, time, and money, increased pollution, and threats to safety. Highways especially are preferred for traveling at high speeds between distant locations. But today the highway infrastructures tend to remain behind the increase in the number of cars traveling on them. As public authorities fail to build new highways fast enough, congestions start to appear on the already existing ones. Previous studies showed that building new highways is not the answer to solving congestions $[1,2]$, mainly because of the costs involved. Also the attractiveness of new routes just attracts more vehicles. In this paper we propose the intelligent lane reservation system for highway(s) (or ILRSH) system designed to increase the efficiency of already existing highways.

ILRSH implements a system for "lane reservation." We propose reserving a lane of the highway for cars traveling on high speeds. Drivers wanting to travel on this lane have to pay a small fee to reserve an empty slot for a specific portion on that lane. In exchange, they are guaranteed a congestionfree travel on the highway. The lane is electronically managed by ILRSH, using sideway sensors, and mobile devices in cars communicating with a control server.

In this we present an extended version of the position paper first presented by Ravi et al. [3]. The authors describe the idea of using a reserved lane on the highway, together with a possible intelligent traffic control (ITC) system designed to manage it. They mention the components of such a system, present the (not so small) problems that have to be solved, and provide several preliminary solutions. In this we explore further the proposal and give concrete solutions to previous problems. We propose algorithms designed to support the systems. We validate our ideas using modeling and simulation, and we present results to sustain the feasibility of the proposed system.

The idea of implementing and enforcing an intelligent lane reservation system is novel and little explored. Lane reservation shares some goals with congestion pricing [4]. Congestion Pricing aims at equitable distribution of traffic across highways in order to handle congestion [5]. This is achieved by setting toll prices for different highways in proportion to the level of congestion in order to encourage drivers to travel on less congested roads. ILRSH also aims 


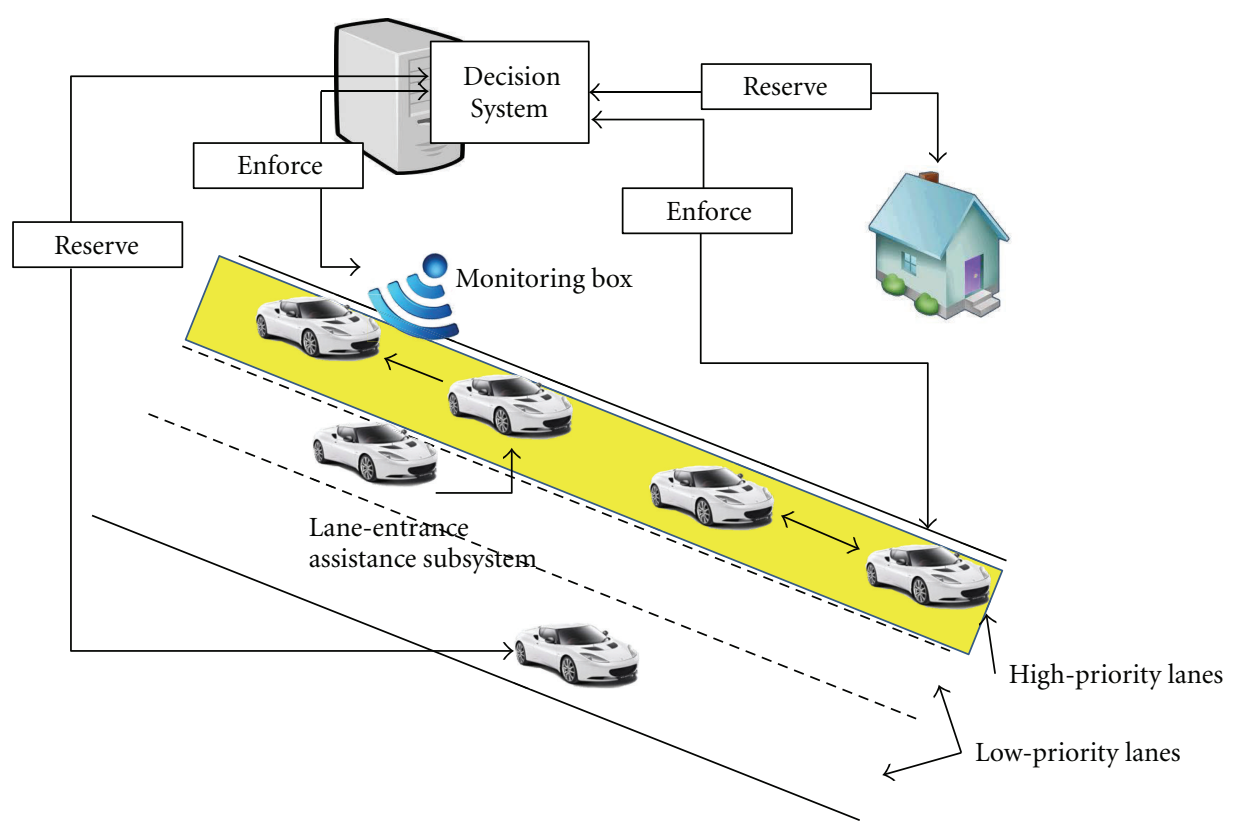

FIGURE 1: System overview. The figure illustrates the three major components of ILRSH: (i) reservation and decision system, (ii) lane entry assistance system, and (iii) enforcement system. Additionally, the various system interactions are shown.

at handling congestion by prioritizing certain lanes on the highway. However, unlike congestion pricing which attempts to address the congestion of a roadway in a uniform manner, we propose differentiating services by introducing highpriority lanes to the roadway.

ILRSH includes several components. Because cars travel at high speeds, we propose components to electronically assist drivers to entry and exit the lane with minimum disruption on already-existing traffic. Special algorithms are designed to assist the drivers take safe decisions for entering and exiting the highway and/or the reserved lane. ILRSH, through its proposed components, is able to automatically manage reservations, and traffic management. As an extent, various ILRSH system can be interconnected and can communicate with each other in order to achieve higher goals: provide multileg reservations (routes spanning multiple highways), cancel/delay reservations for highway $\mathrm{H}_{1}$ in case of a major incident on highway $\mathrm{H}_{2}$, and so forth. Having one ILRSH for each highway simplifies maintenance and provides a clear jurisdiction for each ILRSH. From a software design view, the ILRSH system's design (and implementation) can provide a clear set of services and methods that can also be used by other services/companies (it could support a public interface, in the form of an application programming interface or a web service). From an economical approach, because different ILRSHs would be managed by different companies, this can further generate competition and improvements in the management and scheduling algorithms in order to increase revenue. This will lead to higher quality of service, higher efficiency in using the reserved lane, lower prices for slots, and so forth. So ILRSH has important economic and social implications that benefit, in the end, the drivers as well as the public authorities.
The rest of this paper is structured as follows. In Section 2 we present the architecture of ILRSH. This is followed by the description of the proposed solutions for the reservation and monitoring components. They are at the foundation of the proposed algorithms for the lane entry assistance and the lane exit assistance components, presented next. In Section 8, we present evaluation results obtained using modeling and simulation. Section 9 gives conclusions and presents future work.

\section{System Architecture}

The general architecture of ILRSH is presented in Figure 1. The system includes several components: (1) a reservation and decision subsystem, (2) a lane entrance subsystem, and (3) an enforcement subsystem. The driver can reserve a slot on the reserved lane through the lane reservation subsystem. This subsystem includes a decision component, responsible with the scheduling of traffic slots, depending on the requests versus current traffic conditions. It uses a monitoring subsystem, which is responsible for preserving a correct model of the real-time traffic. The monitoring information is obtained either from sensors available within the highway infrastructure, but also from the wireless devices available in cars (the driver can monitor its current speed and feed-it back to the system, in return possibly negotiating smaller travelling fees). The monitoring sub-system also serves the lane-entrance subsystem (to support the decision of letting the driver enter/exit the reserved lane), and also the enforcement subsystem. The latter is used to control which cars are allowed and or not to travel on the reserved lane. If this subsystem detects a car on the reserved lane that did not pay the reservation fee, it could alert the competent 


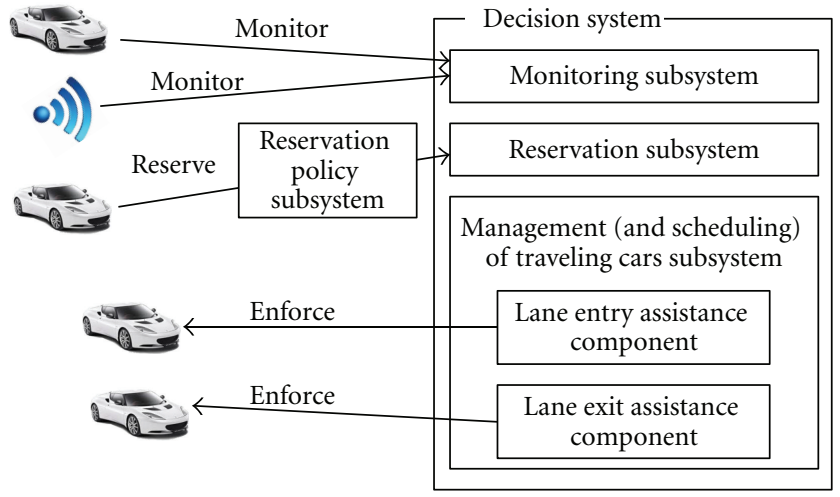

FIGURE 2: The architecture of the ILRSH components.

authorities which, in turn, could result in a small fine applied to the driver.

In what follows we describe the design options for all these components. The reservation subsystem is served by an online service, invoked by the user either from his/her home PC (for advance booking), or from a mobile device (for on-spot reservations the driver can use his smartphone, or for the effective implementation of the proposed system any navigation device can be used). The lane entrance assistance system is based on vehicle-to-vehicle communication.

The decision system itself is composed of several logical components represented in Figure 2. The Monitoring subsystem is responsible for receiving context information from a set of monitoring boxes (sensors on the road level, monitoring the cars) and from the cars themselves (sending their positions).

The reservation subsystem is responsible for the management of the reservations. It takes decisions to accept or reject a request for a reservation, based on whether an empty slot is available (in case of requests for reservations specifying a certain interval of time in the future) or on the occupancy percentage of the reserved lane at current time and the number of valid reservations in the near future (for adhoc reservations). The component uses the services provided by an optional reservation policy subsystem, which implements the pricing policy (the policy regarding the fees that has to be paid by drivers for using the reserved lane), based for example on estimations of the profit made by the company managing ILRSH. Finally, the intermediary between the reservations and current road conditions is the management (and scheduling) of traveling cars subsystem. This component is responsible with monitoring and instructing drivers (in forms of suggestions that they receive ad hoc on their mobile phones) on how to modify their speeds in order to make the best usage of the reserved lane. All these components, together with their functions, are described in details in the next sections.

For ILRSH, we made several assumptions. The reserved lane is considered the leftmost lane for the countries where cars are driven on the right side (and the rightmost lane for the countries where cars are driven on the left side), because that is the lane with the greatest speed limit on highways.
The points where the highway is connected with an entry or exit road are called entry and exit connections.

The cruise speed (CruiseSpeed) on the reserved lane is considered to be $150 \mathrm{~km} / \mathrm{h}$, a bit higher than the allowed maximum speed $(\approx 120-140 \mathrm{~km} / \mathrm{h})$ in some countries. When the local law requires the use of a lower cruise speed, the system is still able to perform well (in fact our simulation experiments show that lower speeds help cars to faster reach the speeds required to enter safe on the lane). Also, the minimum safety distance $\left(D_{\text {min-car-car }}\right)$ between two consecutive cars traveling at CruiseSpeed is set by the local authorities. In our experiments we considered a safety distance of $100 \mathrm{~m}$. This is different than the recommended distance between two cars traveling at top speed, which was set to $D_{\text {car-car }}=125 \mathrm{~m}$. These parameters are important as they affect the maximum capacity of the reserved lane.

We also made several assumptions about cars. First, a car traveling on the reserved lane must be able to reach a top speed of at least $16 \mathrm{~km} / \mathrm{h}$ above the CruiseSpeed. This is because the system may require the driver, at some point, to drive faster in order to better arrange the cars traveling on the reserved lane. Also, we assumed an average car acceleration (considering the technical characteristics of several considered car models). For a smooth acceleration (to maintain the comfort of the passengers inside the car), we considered 15 seconds to be the time needed for the car to reach $100 \mathrm{~km} / \mathrm{h}$ starting from 0 . In our experiments we considered an acceleration of $1.85 \mathrm{~m} / \mathrm{s}^{2}$. We considered similar values for the car's deceleration.

We also state that several ILRSH can be interconnected, and exchange information with each other to achieve higher goals: (1) provide multi-leg reservations, and (2) cancel/delay reservations for highway $\mathrm{H}_{1}$ in case of a major incident on highway $\mathrm{H}_{2}$ (in the event of an incident, cars pass very slow on $\mathrm{H}_{2}$, so the ones having a reservation on $\mathrm{H}_{1}$ shall obviously be late or not able to make it at all).

Having one ILRSH for each highway simplifies their maintenance and provides a clear jurisdiction for each ILRSH. Supposing the system is applied on highways in a specific geographic area, all ILRSH are able to work as a distributed system. From a software design view, having one ILRSH for each highway, forces the ILRSH design (and implementation) to provide a clear set of services and methods that can also be called by other services/companies (i.e. a car renting company would also be able to provide a reservation for a hurried customer for a specific route).

From an economic point of view, because various ILRSH can be managed by various companies, the system can generate competition (just as between airlines, mobile phone operators, or Internet service providers), and improvements in the management and scheduling algorithms to increase revenue.

\section{The Reservation Subsystem}

The reservation subsystem is responsible for matching a free slot (empty space between two consecutive cars traveling on the reserved lane) with a reservation request, on a specific 
time interval. The free slot is a place that can be occupied by a car on the reserved lane, for a specified section of the highway. As mentioned, the relative distance between slots is approximately $D_{\text {car-car }}$, and the relative time is $T_{\text {car-car }}$, considering that cars travel at CruiseSpeed. A slot is valid from one entry point to another exit point (or connections on the highway, from where the car enters the highways until where it wants to exit), for a specific time window. The construction of such a slot depends on the reservation: where the driver wants to start, the required traveling time, and the distance until the desired entry/exit connection. The distance and time difference between slots follows safety regulations (the systems cannot be instructed to "squeeze" a car between two others, if this contradicts safety regulations).

A slot is considered empty on a section of the highway when, for that segment and specific time window, it has not yet been previously reserved. Once a driver reserves an empty slot for a specific section/segment, they are notified on the specific time the slot is centered at. The system allows for a time interval of 15 minutes before that time and 15 minutes after the time for the driver to reach the lane next to the reserved lane and to request to enter the reserved lane.

Reservations can be made from home or a mobile phone connected to the Internet. The user specifies the desired interval of time when will be traveling on the highway, the entry connection and also the exit connection. The reservation subsystem (Figure 2) will search for an empty slot in that interval and will confirm the reservation centered at an exact time. The driver is then notified that he/she can request to enter the reserved lane in an interval of time 15 minutes before that exact time and at most 15 minutes after.

The system also allows adhoc reservations. The reservation subsystem will query the database for an empty slot as close as possible to the current time. If a reservation is found, the system informs the driver about the exact time the reservation is centered at. And it will also call the Lane entry assistance component to start the whole procedure for assisting the driver in entering the reserved lane.

Regarding the reservation policy, Ravi, et al. [3] describes several potential reservation policies. The general architecture of the ILRSH allows a reservation Policy subsystem to be designed and to directly communicate with the Reservation Subsystem. This reservation policy subsystem is important for the company managing the ILRSH since it will generate all its income (and therefore its profit).

\section{Monitoring the Travelling Cars}

The monitoring subsystem uses monitoring boxes within the road infrastructure and mobile applications running on cars. A monitoring box is equipped with sensors (to identify a car) and communications capabilities (to send back the acquired information to the Monitoring subsystem). For sensing these boxes could be equipped, for example, with video cameras. For communication each box could wirelessly send information to an area server (responsible for data collection), which in turns could send the data over wired links to the main monitoring server (such an architecture can lower costs, since each box would be equipped limited hardware).

In the proposed architecture, we use a specific naming convention: the monitoring boxes contain the same equipment, but have different names to distinguish their functions and placement on highway and side roads (the roads connected with the highway through entry and exit points, a difference used, e.g., by the Reservation subsystem). The first part of the name describes the function of the monitoring box, and the second part is used to represent its road placement (in all figures below).

The "after exit connection positioned on road" $\left(A E x C \_P o R\right.$ ) monitoring box (see Figures 3 and 4 ) is used to confirm that a car has exited the highway. It also records the speed of cars traveling on the exit road, and in case this speed goes below a specific limit, the monitoring Subsystem may conclude that the exit connection is jammed. It is positioned at a $D m b \_A E x C \_P o R$ distance after the end of the exit connection. The AExC_PoH monitoring box is used to confirm that a car has exited the reserved lane.

The "after exit connection, positioned on (the reserved lane of the) Highway" ( $A E x C P O H)$ monitoring box is used to confirm that a car has exited the reserved lane, and to provide a clear image of all cars passing on the reserved lane. It is positioned at a $\mathrm{Dmb} \_$ExC $\mathrm{PoH}$ distance after the end of the exit connection.

Another monitoring box could be the "after entry connection positioned on (the reserved lane of the) Highway" $\left(A E n C \_P o H\right)$ one, but (as presented in Figures 3 and 4) this can be omitted. In fact, the system does not need to know what cars are on the reserved lane shortly after the entry connection-the reserved lane entrance algorithm (described below) is not tied to the road from where cars enter. After the entry connection, each car can travel for a while (short or long, depending on where the next free slot starts or is located) on the other lanes (just like the rest of cars), until it is allowed to enter the reserved lane. The same logic applies for a "before exit Connection, Positioned On (the reserved lane of the) Highway" ( $\mathrm{BEx} C \mathrm{PoH})$ monitoring box. There is no need for the system to monitor what cars are on the reserved lane shortly before the exit connection because drivers may leave the reserved lane (at various distances) before the exit connection (some drivers may decide to leave the reserved lane sooner, others shortly just before the exit connection). The important information is what cars are travelling on the reserved lane after the exit connection, which is handled by the $A E x C \_P o H$ monitoring boxes.

The "before entry connection positioned on road" (BEnC_PoR) monitoring box is used by the Monitoring subsystem to control what (and how many) cars are entering the highway. It records the speeds of cars traveling on a particular entry road. In case the average speed decreases below a limit, the Monitoring subsystem may decide that the entry connection is jammed or working very slow. Therefore, the decision subsystem can use this information and compute or predict when to expect cars with valid requests to enter the reserved lane. Such monitoring boxes are also useful for implementing speculative execution algorithms for 


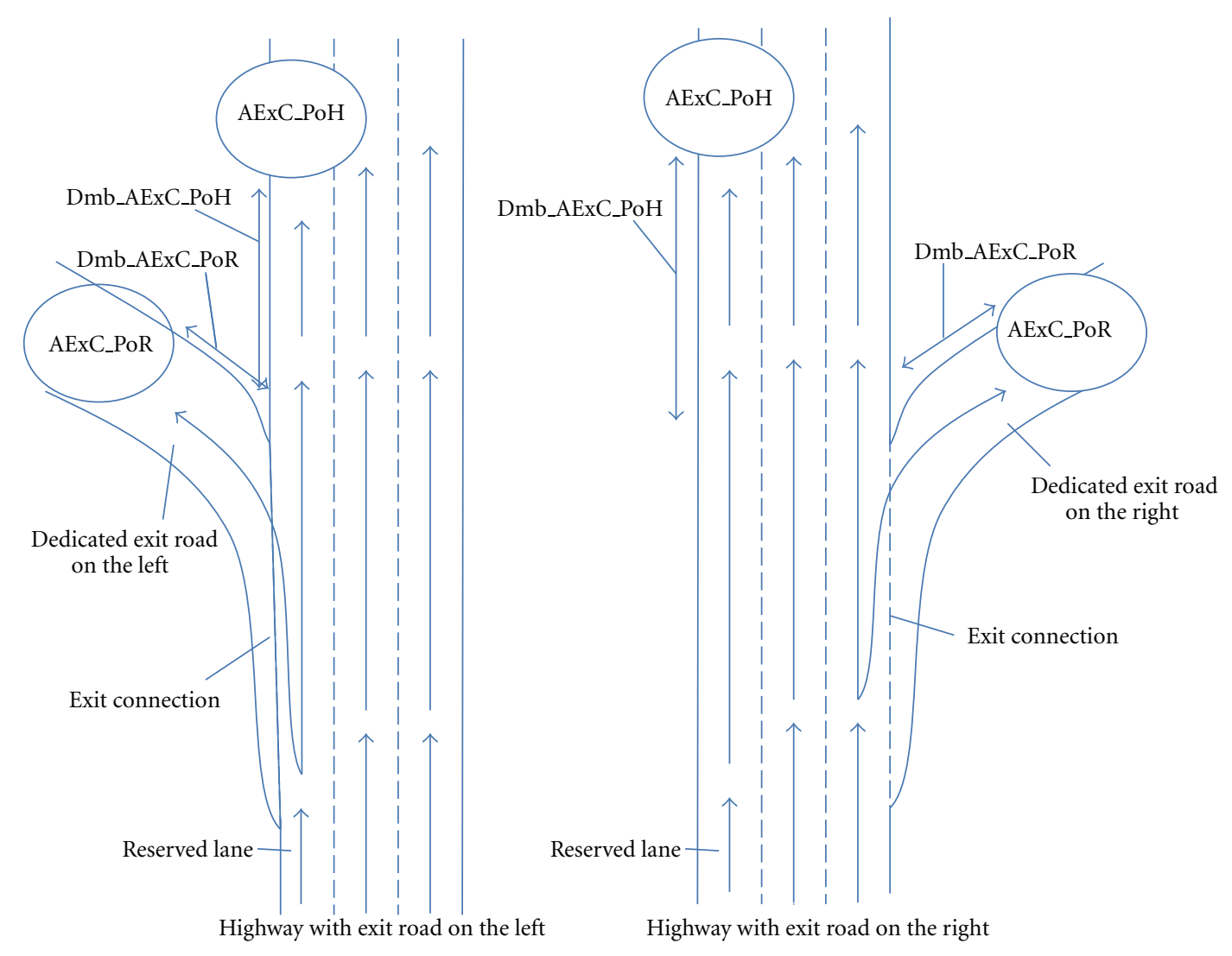

FIgUre 3: Representation of positions of AExC PoR and AExC PoH monitoring boxes.

the management of traveling Cars subsystem (part of the Decision subystem) to better arrange the traveling cars. The monitoring box is positioned at a $\mathrm{Dmb} B E n C$ P PoR distance before the beginning of the entry connection (see Figure 5).

The "in-between positioned on (the reserved lane of the) highway" (IB_PoH) monitoring box monitors the cars passing on the reserved lane. Most of the monitoring boxes placed on the (reserved lane of the) highway will be of this type. They will be placed at a maximum distance of Dmb_IB_PoH from any other monitoring box positioned on the (reserved lane of the) highway.

The monitoring information includes the speeds of the cars passing through (this information is used by the monitoring subsystem to verify if a particular entry or exit connection is congested), and possible the identifiers of the cars passing by. The identifiers are used by the system to control when a car enters (using the BEnC_PoR monitoring boxes) or exits the highway (using the AExC_PoR monitoring boxes). From a security perspective the privacy of drivers is still preserved, the system could use virtual identifiers, disregarding the driver's identity, constructed in turn by the reservation subsystem.

In addition to the monitoring boxes, the monitoring system also relies on cars traveling on the highway to construct an accurate real-time model of traffic conditions. Cars can send data to the monitoring subsystem when entering the wireless communication range of the monitoring boxes. They can broadcast monitoring information back to these boxes, including their identifiers and current GPS-based locations (and travelling speeds). But the system can also function even in the absence of car's information. The monitoring boxes could, for example, be responsible with recognizing the cars. But when cars are used the monitoring information becomes more accurate, and can be used to increase the fidelity of the traffic model. In return, when cars participate in the sending process, they can receive incentives in the form of reduced negotiated fees to enter the reserved lane.

When a car at the entry on the highway receives broadcast information from a monitoring box, it will query the Monitoring subsystem whether that monitoring box is valid or not. Cars ignore by default the information they receive from monitoring boxes they are not expecting. To be considered expected, the box must either be "the next valid one" according to the broadcast information of the last valid box, or be "the next valid one" when the car queried the monitoring subsystem.

We also propose several solutions for placing the monitoring boxes. A first possible solution envisions the monitoring boxes placed on the highway and on side roads (at the connection with the highway). This solution uses all types of monitoring boxes. In this approach, the monitoring 


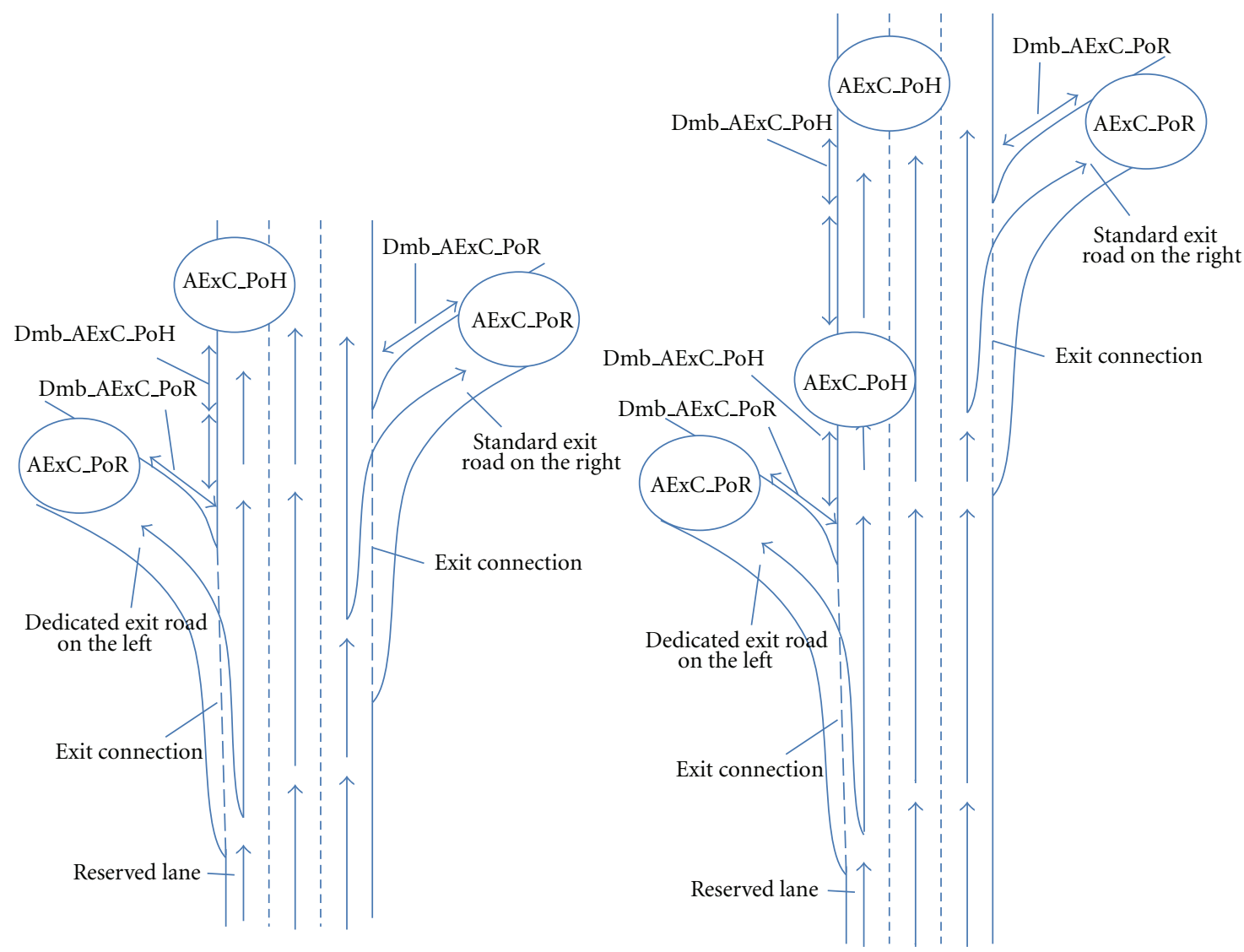

FIgURE 4: Representation of positions of AExC_PoR and AExC_PoH monitoring boxes for close and not close exit connections. Left is the case of a highway with both types of exists, placed close to each other, right for highway with both types of exits, placed too far from each other.

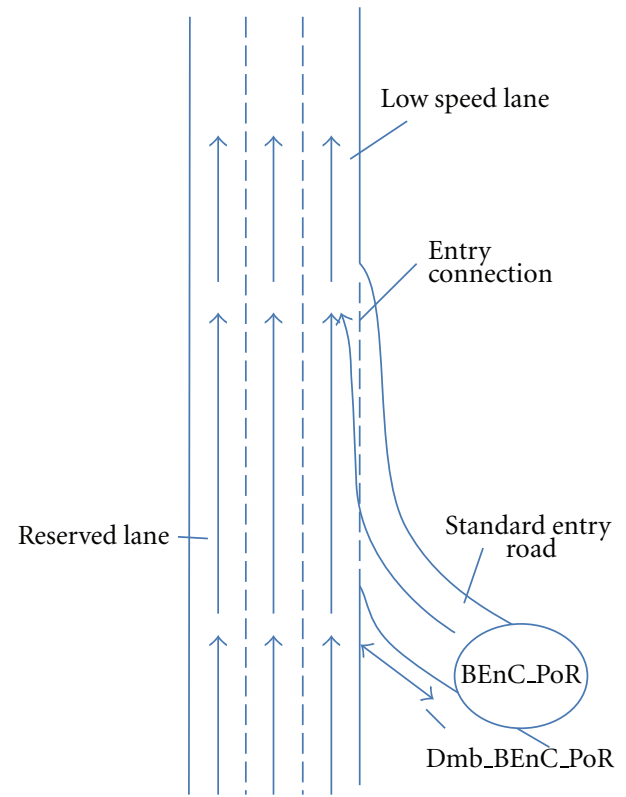

FIGURE 5: Representation of positions of BEnC_PoR monitoring boxes. boxes will be placed as previously described. The monitoring process will not require cars to send monitoring information, because it relies only on the information sent by the monitoring boxes.

An alternative approach consists in the use of only a few monitoring boxes combined with the information (position, time) sent by cars. Such a solution uses the AExCPoH monitoring boxes to send broadcast requests for cars to report data when they are within wireless communication range. The system monitors the status of the reserved lane by relying on the information sent by cars. When a car passes through a monitoring box, it sends its identifier and current position. In this case a $100-150 \mathrm{~m}$ distance can provide a good resolution and not flood the system with information sent by cars. The issue of trusting the data received from cars can be resolved using the approach we previously explored in [6].

Finally, a third solution combines the first and second approaches. First, using the monitoring boxes, the system receives monitoring data from its own equipment. Second, the cars are reporting as they pass the monitoring boxes. This offers a fine resolution for the whole system on the actual situation on the reserved lane. The comfortable number of monitoring boxes assures against also any "hacker drivers." 


\section{Explored Solutions of Decelerating and Accelerating the Cars}

Several solutions are proposed for advising the drivers when to decelerate and then accelerate in a controlled and stable manner to allow the safe entrance on the reserved lane of another car. The solutions presented in this Section are used by the lane entry assistance component of the proposed system.

We first introduce several concepts. The absolute deceleration position $(A D P)$ is a point where the platoon of passing cars must start a deceleration process with a reference deceleration. By platoon we do not mean that cars are automatically assisted in following each others, but we use the term to denote a queue of cars driving together along the reserved lane in a formation, a line, filling up as best as possible (as instructed by our proposed system) the spaces on that lane. The absolute acceleration position $(A A P)$ indicates where cars should start accelerating with a reference acceleration. Also, car $_{1}$ is the first car reaching the ADP, and $\operatorname{car}_{n}$ the last car that is affected by ADP. Cars following/after $\operatorname{car}_{n}$ keep driving with CruiseSpeed. The acc and $\mathrm{dec}$ are the values for the reference acceleration and reference deceleration (values are equal).

We present a first solution called the decelerated platoon. It works as follows. At $t=t_{y, \mathrm{ADP}}$, the $\mathrm{car}_{y}$ reaches ADP and starts decelerating. At $t=t_{y+1, \mathrm{ADP}}=t_{y, \mathrm{ADP}}+T_{\text {car-car }}, \mathrm{car}_{y+1}$ reaches ADP after $T_{\text {car-car, }}$, and starts decelerating. When $\operatorname{car}_{y+1}$ reaches ADP, car $_{y}$ would have gone a distance equal to

$$
\Delta x_{y, y+1}\left(t_{y+1, \mathrm{ADP}}\right)=\text { CruiseSpeed } \cdot T_{\text {car-car }}-\frac{\mathrm{dec} \cdot T_{\text {car-car }}}{2} .
$$

Because $\operatorname{car}_{y}$ and $\operatorname{car}_{y+1}$ decelerate with the same deceleration, $\Delta v$ is preserved as follows:

$$
\begin{aligned}
\Delta v_{y, y+1}\left(t_{y+1, \mathrm{ADP}}\right) & =v_{y}\left(t_{y+1, \mathrm{ADP}}\right)-v_{y+1}\left(t_{y+1, \mathrm{ADP}}\right) \\
& =\text { CruiseSpeed }-\mathrm{dec} \cdot T_{\text {car-car }}
\end{aligned}
$$

Since car $y$ and $\operatorname{car}_{y+1}$ start decelerating when they reach the same ADP and accelerate at the same AAP, the time between them is preserved. In this case $\operatorname{car}_{y+1}$ reaches the same position and speed as car $y$ at exactly $T_{\text {car-car }}$ after car $_{y}$.

$$
\Delta v_{y}\left(t_{y, \mathrm{AAP}}\right)=\text { CruiseSpeed }-\mathrm{dec} \cdot \tau_{y, \mathrm{ADP}-\mathrm{AAP}}
$$

where $\tau_{y, \mathrm{ADP}-\mathrm{AAP}}=\left(\right.$ CruiseSpeed $\left.-v_{y}\left(t_{y, \mathrm{AAP}}\right)\right) / \mathrm{dec}$.

Because $v_{y}\left(t_{y, \mathrm{AAP}}\right)$ is the same for all $\mathrm{car}_{y}$, we can use:

$$
\tau_{\mathrm{ADP}-\mathrm{AAP}}=\tau_{y, \mathrm{ADP}-\mathrm{AAP}}=\tau_{y+1, \mathrm{ADP}-\mathrm{AAP}} .
$$

At $t=t_{y, \mathrm{AAP}}+\tau_{y, \mathrm{ADP}-\mathrm{AAP}}=t_{y, \mathrm{ADP}}+2 \cdot \tau_{\mathrm{ADP}-\mathrm{AAP}}$, $\operatorname{car}_{y}$ reaches CruiseSpeed. At $t=t_{y+1, \mathrm{AAP}}+\tau_{y+1, \mathrm{ADP}-\mathrm{AAP}}=$ $t_{y, \mathrm{ADP}}+2 \cdot \tau_{\mathrm{ADP}-\mathrm{AAP}}+T_{\text {car-car }}, \mathrm{car}_{y+1}$ reaches CruiseSpeed. So the distance between these cars is

$$
\Delta x_{y, y+1}=\text { CruiseSpeed } \cdot T_{\text {car-car }}=D_{\text {car-car }} \text {. }
$$

In conclusion, the distance between cars is preserved. The solution has the advantage that after cars reach CruiseSpeed again, the initial configuration is fully restored. Slowing down car $_{1}$ has created a larger distance between it and the car in front of it, which is recovered by slowing down cars once they reach ADP (and accelerating them to CruiseSpeed after AAP).

To delete the decelerated platoon, we first explored the alternative of suddenly deleting the ADP. The problem is that $\operatorname{car}_{n}$ travels at a speed that keeps decreasing, while $\operatorname{car}_{A n}$ continue traveling at CruiseSpeed. In this case at $t=$ $t_{n, \mathrm{ADP}}, \operatorname{car}_{n}$ starts to decelerate. At $t=t_{n, \mathrm{ADP}}+T_{\text {car-car }}, \operatorname{car}_{A n}$ reaches ADP, but still drives at CruiseSpeed, while $\operatorname{car}_{n}$ still decelerates. At $t=t_{\text {warning, }}, \operatorname{car}_{n}$ and $\operatorname{car}_{A n}$ reach the smallest allowed distance (for safety reason), which is $D_{\text {mincar-car }}$. Considering $\Delta t=t_{\mathrm{warning}}-t_{n, \mathrm{ADP}}$, it is easy to see that

$$
D_{\text {car-car }}-\frac{\mathrm{dec} \cdot(\Delta t)^{2}}{2}>D_{\text {min car-car }}
$$

(because the rule $\Delta x_{n, A n}>D_{\text {mincar-car }}$ must apply), which results in:

$$
\sqrt{\frac{\left(D_{\text {car-car }}-D_{\text {min car-car }}\right) \cdot 2}{\text { dec }}}>\Delta t .
$$

For ILRSH's considered parameters, this means that the maximum allowed value is $\Delta t \approx 5.2 \mathrm{~s}$. Otherwise, in the best case $\operatorname{car}_{A n}$ will get to close to $\operatorname{car}_{n}$.

Such a small time is insufficient for a safe implementation of the proposed system. We next turned to an alternative solution, where the ADP is allowed to move towards AAP with a speed $v_{\mathrm{ADP}}$, right after the first cars in the platoon passes by. When ADP reaches AAP, the platoon is basically deleted. AAP, ADP and $v_{\mathrm{ADP}}$ can be computed from the beginning, based on the target low speed $V$ that car $_{1}$ needs to have when it reaches AAP.

The formulas again consider the generic case of two cars, $\operatorname{car}_{y}$ and $\mathrm{car}_{y+1}$, traveling on the reserved lane. At $t=t_{y, \mathrm{ADP}}$, car $_{y}$ reaches ADP. At $t=t_{y+1, \mathrm{ADP}}=t_{y, \mathrm{ADP}}+T_{\text {car-car }}+t_{d}, \mathrm{car}_{y+1}$ reaches ADP. Between the two moments ADP travels the $x_{d}$ distance.

Because ADP moves with $v_{\mathrm{ADP}}$, the distance $x_{d}$ decreases starting from the initial distance between ADP and AAP, denoted by $d_{\mathrm{ADP}-\mathrm{AAP}}$. The two cars will travel different distances:

$$
\begin{gathered}
d_{y, \mathrm{ADP}-\mathrm{AAP}}=d_{\mathrm{ADP}-\mathrm{AAP}}-x_{d} \cdot(y-1), \\
d_{y+1, \mathrm{ADP}-\mathrm{AAP}}=d_{y, \mathrm{ADP}-\mathrm{AAP}}-x_{d}=d_{\mathrm{ADP}-\mathrm{AAP}}-x_{d} \cdot y .
\end{gathered}
$$

At time $t=t_{y, \mathrm{AAP}}=t_{y, \mathrm{ADP}}+\tau_{y, \mathrm{ADP}-\mathrm{AAP}}$, car $_{y}$ reaches AAP. The $\tau_{y, \mathrm{ADP}-\mathrm{AAP}}$ is the required time for $\mathrm{car}_{y}$ to travel between ADP and AAP. Consider

$$
\begin{gathered}
v_{y}\left(t_{y, \mathrm{AAP}}\right)=\sqrt{\text { CruiseSpeed }^{2}-2 \cdot \mathrm{dec} \cdot d_{y, \mathrm{ADP}-\mathrm{AAP}}} \\
\tau_{y, \mathrm{ADP}-\mathrm{AAP}}=\left(\text { CruiseSpeed }-v_{y}\left(t_{y, \mathrm{AAP}}\right)\right) / \mathrm{dec} .
\end{gathered}
$$

At time $t=t_{y+1, \mathrm{AAP}}=t_{y+1, \mathrm{ADP}}+\tau_{y+1, \mathrm{ADP}-\mathrm{AAP}}, \mathrm{car}_{y+1}$ reaches AAP. Consider

$$
v_{y+1}\left(t_{y+1, \mathrm{AAP}}\right)=\sqrt{v_{y}^{2}\left(t_{y, \mathrm{AAP}}\right)+2 \cdot \mathrm{dec} \cdot x_{d}} .
$$




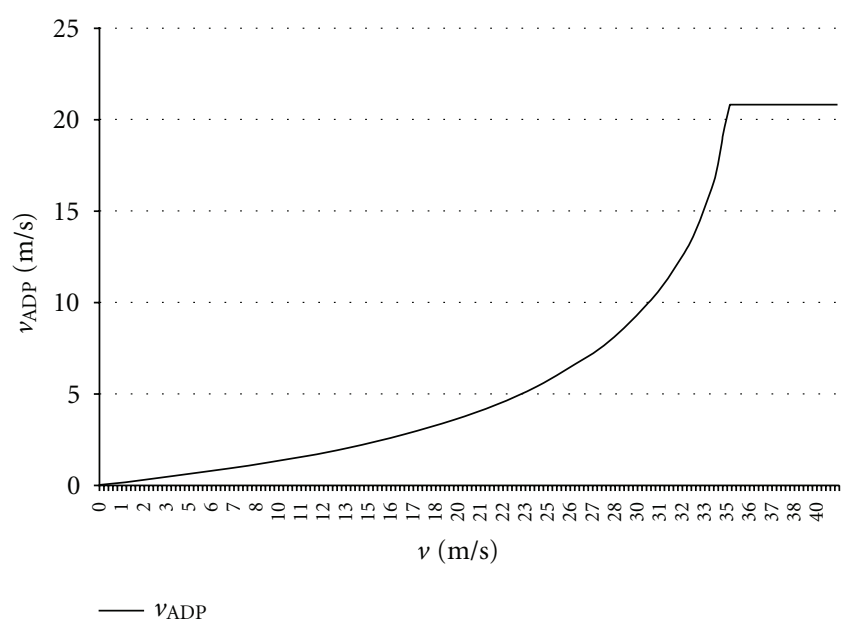

FIGURE 6: Values of $v_{\text {ADP }}$ for different values of $V$.

Note that

$$
v_{y+1}\left(t_{y+1, \mathrm{AAP}}\right)>v_{y}\left(t_{y, \mathrm{AAP}}\right)
$$

which means the low speed cars reach AAP at increased speeds (cars with higher $y$ index will have a higher low speed when they reach AAP).

Also because

$$
\tau_{y+1, \mathrm{ADP}-\mathrm{AAP}}=\frac{\left(\text { CruiseSpeed }-v_{y+1}\left(t_{y+1, \mathrm{AAP}}\right)\right)}{\operatorname{dec}},
$$

we get

$$
\tau_{y+1, \mathrm{ADP}-\mathrm{AAP}}<\tau_{y, \mathrm{ADP}-\mathrm{AAP}},
$$

which means cars take less time to travel between ADP and AAP.

By continuing this logic, it is easy to see that the smallest distance between cars is when $y=1$. This is why the parameters are computed so that the minimum distance $\Delta x_{1,2}=D_{\text {min car-car }}$.

The implementation of the proposed algorithm is described in Algorithm 1.

Figure 6 gives a good representation of all values $v_{\mathrm{ADP}}$ can take. We also present in a subsequent section more results for several computed parameters of the decelerated platoon, constructed for various values of $V$.

\section{The Lane Entry Assistance Component}

Since cars travel on the reserved with $150 \mathrm{~km} / \mathrm{h}$, consecutive cars pass a fixed observation point every $3-4$ seconds. It is difficult for a driver to observe where a free slot is, and enter the reserved lane and reach the CruiseSpeed. The lack of automated assistance can, therefore, lead to accidents (the worst case) and great traffic disruptions (the best case scenarios). So, a lane entry assistance component (LEAC) is needed to assist both the entering car and the already traveling on the reserved lane cars.
6.1. Rules of Assisting the Entrance the Reserved Lane. A car can be assisted to enter the reserved lane after it has traveled a distance of at least $D_{\text {aftermB }}$ after any valid $A E x C P O H$ monitoring box or, in case the $A E x C_{-} P o H$ is broken, after a valid $I B \_P o H$ monitoring box. $D_{\text {afterMB }}$ is equal or greater than the RD computed by the LEAC's algorithm when the car requests to enter the reserved lane.

A car is not allowed to enter the reserved lane $200 \mathrm{~m}$ prior to the exit connection (for a car traveling at $150 \mathrm{~km} / \mathrm{h}$ this means roughly 5 seconds; this is a sensitive time interval when the driver prepares to exit the highway and does not need any interference, and it will not pay attention to the system, assuming the system tries to assist another car to enter the reserved lane).

6.2. The Proposed Algorithm. The algorithm is based on the relative speed between cars and will create a "decelerated platoon," inside it cars have lower speeds the closer they are to the empty slot. The new car will occupy the empty slot. Basically, in the first phase, when each car passes by "absolute slowing down position" ASDP it will be required to slow down with the reference deceleration; the distance between the cars in the platoon will decrease. Then, in the second phase, ASDP will be deleted and absolute acceleration point (AAP) will be created; when each of the cars in the platoon passes by the AAP, it will be required to accelerate, and so the initial configuration will be reconstructed (when they all reach CruiseSpeed).

We propose several names for the key cars in this case. $\mathrm{NC}$ - "new car" - is the car that wants to enter the reserved lane and will occupy an empty place there on the reserved lane. AEP_-"after empty place" car-is the car occupying its own slot on the reserved lane, but after the empty place. IFEP_ "in front of the empty place" car-is the car right in front of the empty place, the opposite of AEP.

When the car having a valid reservation enters the lane, next to the reserved lane it can send a request to enter the reserved lane. At this point, the lane entry assistance component becomes aware that the car is traveling with a speed $v_{\text {low }}$ while the cars on the reserved lane travel at the high speed of CruiseSpeed.

The system computes the required distance RD and time RT for a car traveling on the reserved lane to decrease its speed by half of $\Delta v_{\mathrm{CS}-\mathrm{NC}}=$ CruiseSpeed $-v_{\text {low }}$ (this way when AEP has traveled RD, AEP and NC will be traveling on the reserved lane at approximately the same speed). Afterwards, based on the NC's position, it will search for an empty place on the reserved lane (therefore an empty slot) at a distance equal or greater that RD; the LEAC can also "invent" an empty place at half the distance between 2 cars traveling at a regular distance, so it will basically "insert" NC between them, and in the end arrange all of them to respect $D_{\text {car-car. }}$ The system will keep track of that empty place and will position NC on it at the right time; it will send an estimated time to NC on how long it will take that empty place to reach it; it will also send an estimated time to AEP and the cars after it, so these drivers will know how long they have before starting to slow down. 


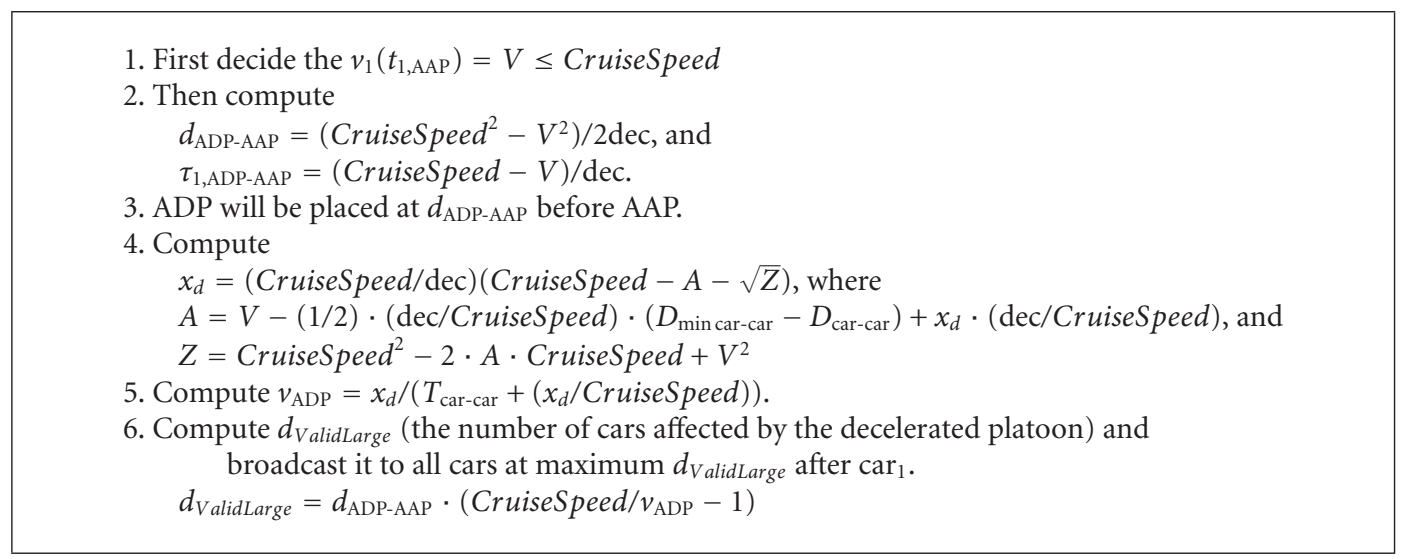

Algorithm 1: The proposed algorithm for decelerated platoon.

After the entry connection, NC will travel for a while on the other lanes until it is allowed to enter the reserved lane. (The entry on the reserved lane can happen almost anywhere, with some restrictions imposed by the lane entrance rules.)

When that empty place is at a distance of RD from NC, that position becomes ASDP. When AEP passes by ASDP, it will receive the signal to slow down with the reference deceleration. When the car occupying the next slot right after AEP passes by ASDP, it will receive the same information, and will start decelerating. Technically, since it occupies right the next slot after AEP, this car will start slowing down after $T_{\text {car-car }}$ seconds (the distance between these cars will decrease in the first phase by $\mathrm{car}_{d} \cdot T_{\text {car-car }}^{2} / 2$, and then every second due to the difference in speeds between them $\Delta v=\operatorname{car}_{d} \cdot T_{\text {car-car }}$ ). After it passes ASDP, the same thing will happen for the next car, but now having as reference the one after the AEP and so on. So, the difference in speed between every 2 cars that occupy consecutive slots in the decelerated platoon is $\Delta v$. The same difference in speed will be between the last car of the platoon and the first one after the platoon.

When IFEP passes by NC, it will signal it to enter the reserved lane; the same signaling will come from the lane entry assistance system in case there is no car right in front of the empty place (i.e., when the reserved lane is free on that portion).

After NC enters the reserved lane, it will start accelerating with at least the reference acceleration. When AEP has traveled RD from ASDP, no car passing by ASDP will start decelerating any longer; the current position of AEP will become AAP and each of the cars in the platoon will start accelerating when they pass by AAP; also, at this point AEP and NC should approximately have the same speed and the distance between them will be $v_{\text {low }} \cdot \mathrm{RT}+\mathrm{car}_{a} \cdot t^{2} / 2$, which is greater than $D_{\text {min car-car }}$ and around $D_{\text {car-car }}$.

When NC reaches CruiseSpeed, there is a greater distance (GD) than $D_{\text {car-car }}$ between it and IFEP; at the same time the last car in the decelerated platoon has a smaller distance (SD) than $D_{\text {car-car }}$ between it and the car right after it. To make up for this, first NC will be brought at around $D_{\text {car-car }}$ of AEP, and then NC and the platoon will be told to drive at a speed of $16 \mathrm{~km} / \mathrm{h}$ greater than CruiseSpeed, so GD will decrease by
$4.47 \mathrm{~m} / \mathrm{s}$ and the SD increases by the same value; they will use CruiseSpeed when GD reaches $D_{\text {car-car }}$.

\section{The Lane Exit Assistance Component}

The reasons for the design of lane entry assistance component (LEAC) are similar to the ones presented for the lane exit assistance component. First, to avoid accidents due to the speed difference, cars on the reserved lane travel at a much higher speed than the speed required when entering the dedicated exit or the speed that cars travel on the lane next to the reserved one. Second, we want to minimize the traffic disruption generated by the exiting car (which is slowing down).

7.1. The Considered Cases for the Exit Connection When the Driver Reaches It. When the driver reaches the desired exit connection, the following possible cases need to be considered.

(i) Ideal case: the desired exit connection is free, so the driver can exit the highway.

(ii) Win-Win case: the desired exit connection is jammed, but the driver is still allowed to drive on the reserved lane (the system still has free slots) until reaches the next exit connection. The driver wins because it will travel fast to the next exit, and the system wins because it will charge the driver for the additional use of the reserved lane.

(iii) "Driver loses" case: when it reaches the exit connection, the driver is forced to exit the reserved lane, although the exit is jammed, because there are no free slots that the system can sell. The driver loses time because they will be forced either to use the low speed lanes until it reaches the next free exit, or stand in lane at the jammed exit connection.

(iv) "Lane loses" case: this approach tries to solve the "driver loses" case by making the reservation Subsystem reserve by default the following next $x$ exits after the one the driver has specified. This way, the system 
knows for sure that the driver will be able to exit the highway using the first free exit connection, from the interval up to the $x$ th implicitly reserved. So, the driver wins. The system loses because the slot starting from the driver's desired exit up to the $x$ th exit is considered reserved, although there is quite a small probability that the driver will go all the way and pay for all; the driver will pay for all just in the case all the exits are jammed until the $x$ th.

(v) "Lane loses" solution based on statistics": We can compute statistics on the occupancy level of each exit connection, on days and hours. Each exit will receive a score reflecting its occupancy level. Based on these statistics, the system will be able to determine whether an exit connection is likely to be jammed or not for the specific time that the driver will use it. If the system decides that the exit is going to be jammed, it will search until the $x$ th exit and find the first exit that it expects to be free. It will tell the driver that it has reserved until the $x$ th exit connection because that is the first exit that the system expects to be free starting from the driver's desired exit connection. The system risks because it will get to charge the driver just like in the "lane loses" case, that is, in the case the driver is forced to travel to another exit than the desired one. However, the system only risks in part, because it expects all those exits until the $x$ th to be jammed, so it is very likely that the driver will drive to the free exit, and the system will get paid. In case that even the $x$ th exit connection is jammed, although it should not have been, ILRSH will try to apply the "win-win" case if possible; if it is not possible, then the "driver loses" case.

7.2. The Algorithm. The lane exit assistance component is somewhat similar to the LEAC (lane entry assistance component), since it uses the same concept of "decelerated platoon."

Let us consider that the exiting car (EC) can exit through an exit connection. If it is a dedicated exit connection, then the exit point is exactly the exit connection. If it is a standard exit connection, then the exit point is somewhere before that exit connection, so EC can exit the reserved lane, enter a low speed lane on the right, and may cross the other lanes until it reaches the exit connection. Both cases require EC to reach a $v_{\text {low }}$ speed until the exit point.

The system will compute the required distance (RD) and required time (RT) for the car to reach $v_{\text {low }}$ at the exit point, considering it decelerates with the reference deceleration. So, at RD before the exit point, the system will define the ASDP (just as in the algorithm for LEAC). All cars, when passing by ASDP, will receive the signal to slow down with the reference deceleration and they will form the "decelerated platoon." Same observations apply here, regarding the decreasing distance between cars and the relative speed $\Delta v$ between cars.

After EC has exited the reserved lane, no cars passing by ASDP will start decelerating any longer; at the absolute position of AEP, the system will create AAP; when each of the cars in the platoon passes by AAP, they will start accelerating and when they all reach CruiseSpeed the initial configuration is reconstructed. Of course, the distance (called SD) between the last car in the platoon and the slot after it is smaller than $D_{\text {car-car }}$, so just like in LEAC all the platoon will be brought at a speed higher than CruiseSpeed until $S D$ reaches $D_{\text {car-car }}$.

\section{Experimental Results}

To evaluate the performance of the proposed solution, we next performed a series of simulation experiments. In these experiments the simulated model consisted of different cars and monitoring boxes. For the presentation of the decelerated platoon, the simulation scenario consists of a platoon of 10 cars. The simulated conditions are the ones assumed and presented in the previous sections (cars on the reserved lane can travel, e.g., at approximately $150 \mathrm{~km} / \mathrm{h}$ ). For the obtained results, the dotted lines are positions of ADP and AAP. Figure 7 shows how the decelerated platoon preserves the distance and time between the members of the platoon. As noticed, the created distance between car $_{1}$ and the car in front of it is recovered by having a decreased distance between the last car of the platoon and the car after it (see Figure 7(c)).

Figures 8 and 9 show that collisions are more likely to occur when $V$ decreases. Of course such an approach cannot work, and this is why we next turned to the decelerated platoon algorithm previously presented. In these figures the "position" is measured in meters. For the decelerated platoon, the two extra horizontal lines that appear are the fixed positions of ADP and AAP that affect the predefined platoon of 10 cars. For the graphics based on the solution of Decelerated Platoon, two extra lines appear: the horizontal line is the fixed position of AAP and the one below is ADP, which starts moving towards AAP once it becomes active (once active, ADP affects cars only until it reaches AAP).

The "speed" is measured in meters/seconds. It shows how the speed of each car modifies over time (CruiseSpeed = $41.67 \mathrm{~m} / \mathrm{s}$ and the cars affected by deceleration record lower speeds); the main goal is to obtain values closer to the CruiseSpeed because that means less disturbance for cars since they have to decelerate less.

Finally, the "distance between cars when they reach CruiseSpeed again" is measured in meters and is measured for every two consecutive cars.

Next we evaluated the lane entry algorithm. We started with $V_{\mathrm{NC}}\left(t_{0}\right)=0.00 \mathrm{~m} / \mathrm{s}$, meaning that $\operatorname{car}_{\mathrm{NC}}$ is stopped on the lane next to the reserved lane, because that lane is jammed. Figure 10 shows how the lane entry algorithm works based on the solution of deceleration/acceleration of cars implemented in the ILRSH.

Figures 11 and 12 show how the lane entry algorithm works. Figure 11 considers the case of $125 \mathrm{~m}$ between all cars, and Figure 12 considers the case of a distance of $250 \mathrm{~m}$ between $\operatorname{car}_{\mathrm{IF}}$ and $\mathrm{car}_{1}$.

As a conclusion, the lane entry algorithm works well. Its performance increases when the chosen solution for deceleration/acceleration of cars inside the ILRSH performs 


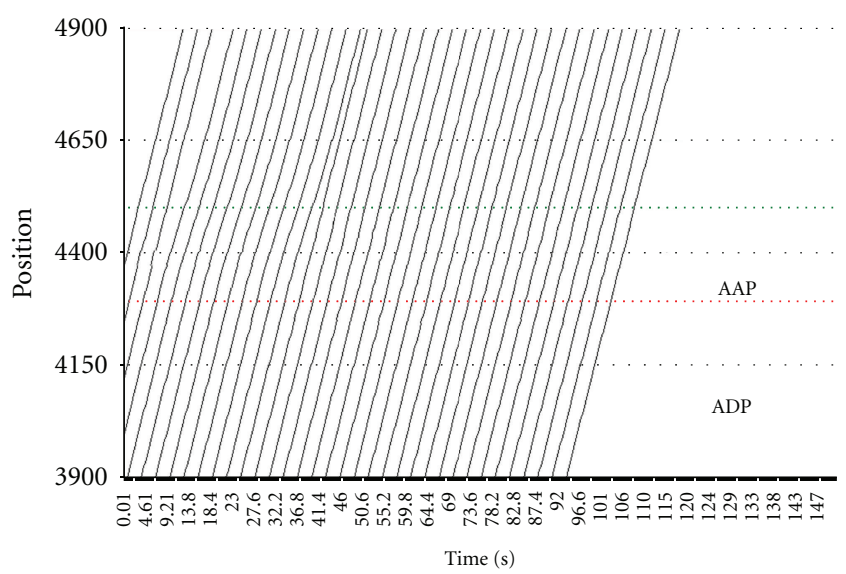

(a) Positions occupied by cars

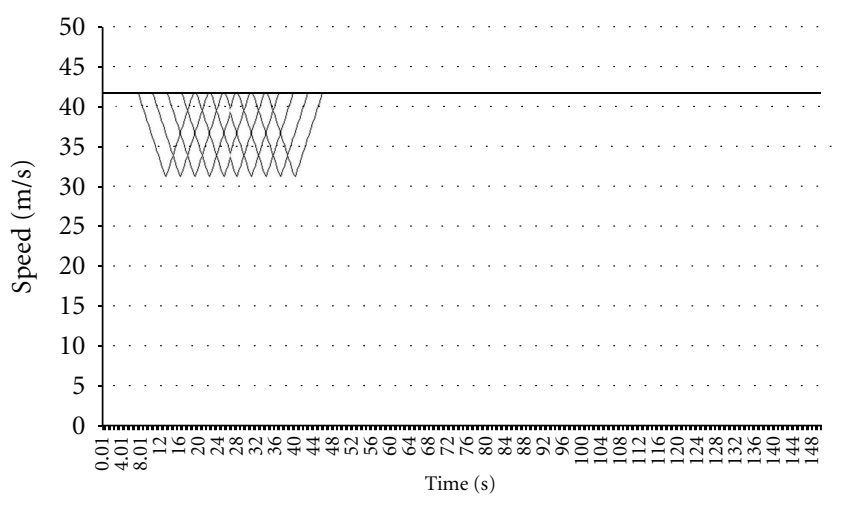

(b) Speed of cars

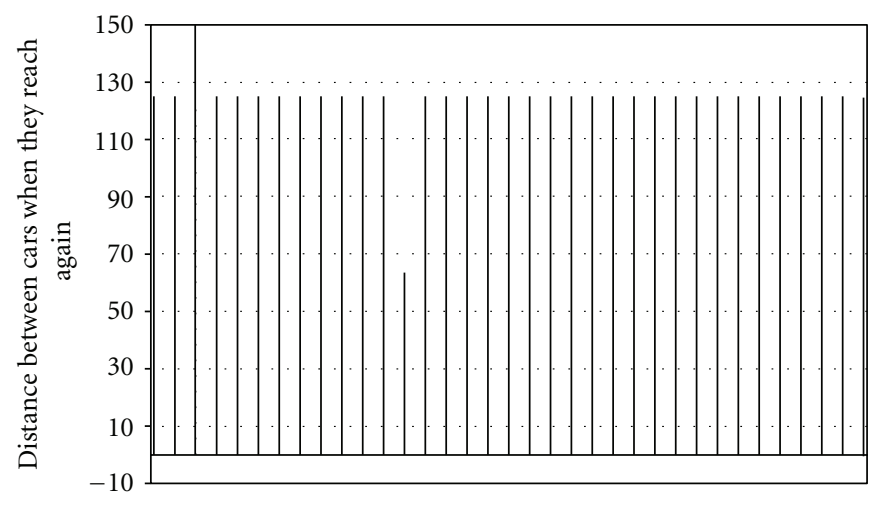

(c) Distances between cars when they all reach CruiseSpeed again

Figure 7: Decelerated Platoon of 10 cars for $V=31.00 \mathrm{~m} / \mathrm{s}$.

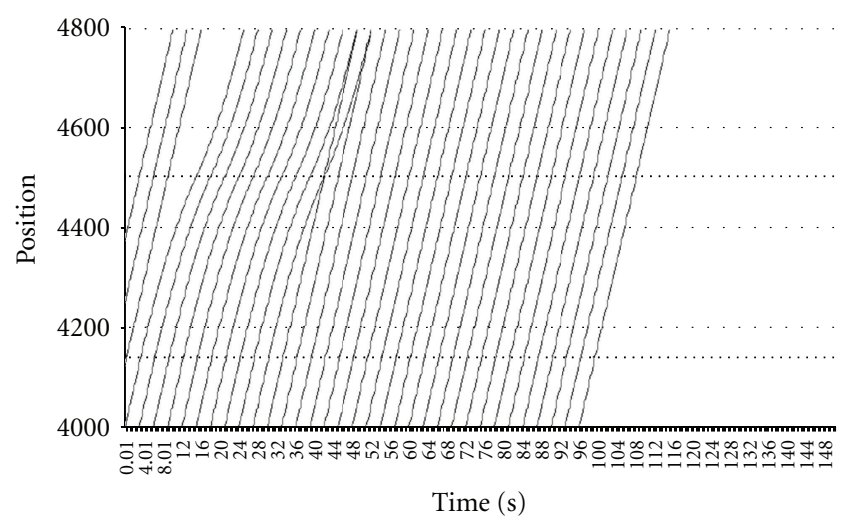

FIgURE 8: The positions occupied by cars for Decelerated Platoon of 10 cars, with $V=20.21 \mathrm{~m} / \mathrm{s} \Rightarrow$ collisions at $t=44 \mathrm{~s}$.

better. This is why for small values of $V_{\mathrm{NC}}\left(t_{0}\right)$ the number of affected cars is also small.

\section{Conclusions}

In this paper, we made a case for prioritizing lanes on highways in order to provide congestion free travel during

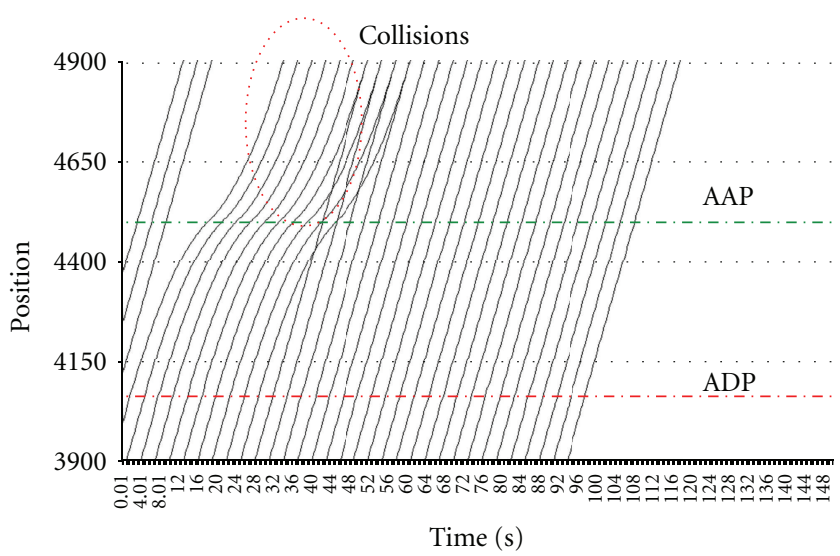

Figure 9: The positions occupied by cars for Decelerated Platoon of 10 cars, with $V=11.15 \mathrm{~m} / \mathrm{s} \Rightarrow$ collisions at $t=41 \mathrm{~s}$.

periods of high volume. We described the design rationale of an intelligent lane reservation system. The system uses a designated lane to support cars traveling at higher speed, if they are willing to pay a small traveling fee. ILRSH implements a system for "lane reservation." We proposed reserving a lane of the highway for cars traveling on high 


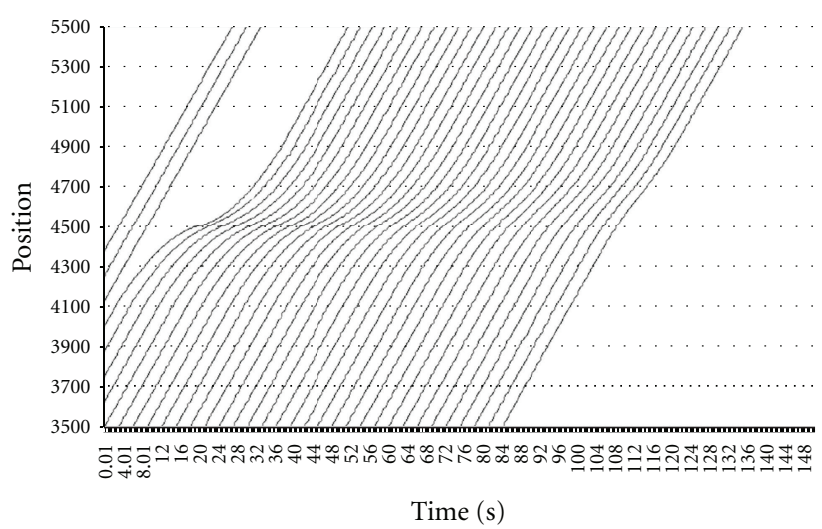

(a) Positions occupied by cars

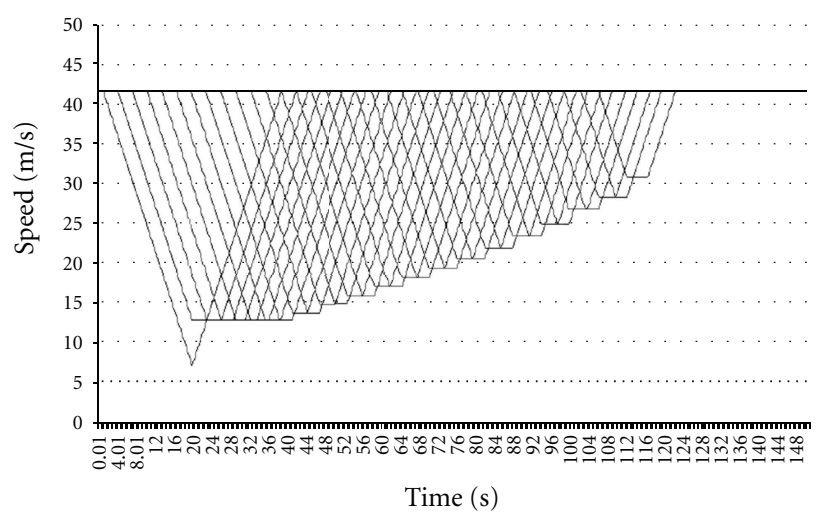

(b) Speed of cars

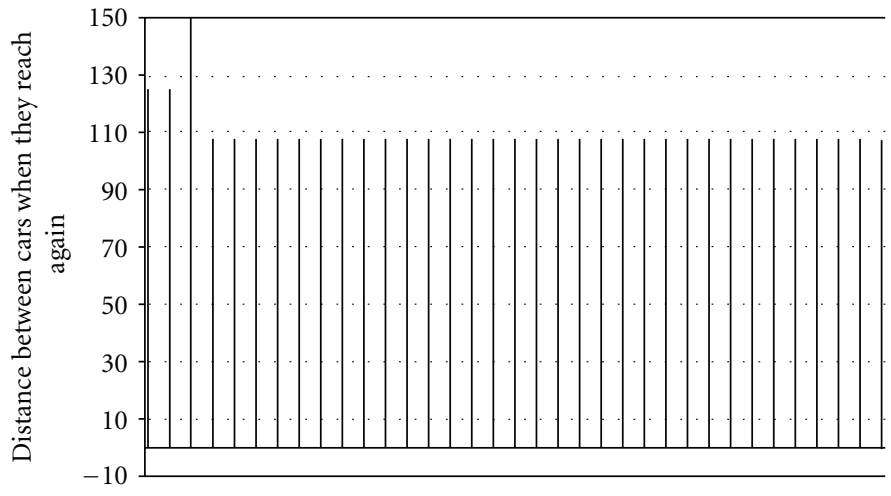

(c) Distances between cars when they all reach CruiseSpeed again

FIGURE 10: The deceleration/acceleration positions; $V=6.90 \mathrm{~m} / \mathrm{s}$; more than 33 cars got affected.

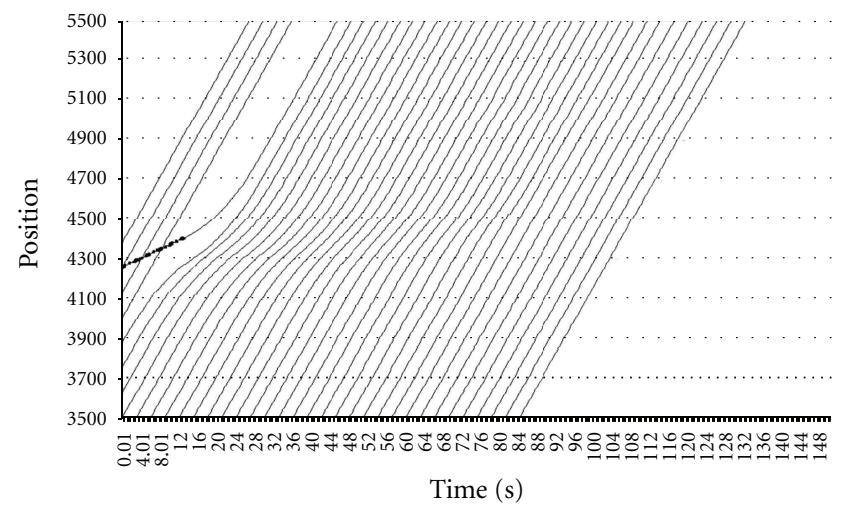

FIGURE 11: The positions occupied by cars in case of the lane entry algorithm, with $V_{\mathrm{NC}}\left(t_{0}\right)=11.15 \mathrm{~m} / \mathrm{s}$; distance between cars $125 \mathrm{~m}$. The thicker line represents $\operatorname{car}_{\mathrm{NC}}$ being on the lane next to the reserved lane; when it becomes a normal line, the car has entered the reserved lane.

speeds. Drivers wanting to travel on this lane have to pay a small fee to reserve an empty slot for a specific portion on that lane. In exchange, they are guaranteed a congestion free travel on the highway. The lane is electronically managed by ILRSH, using sideway sensors, and mobile devices in cars communicating with a control server.

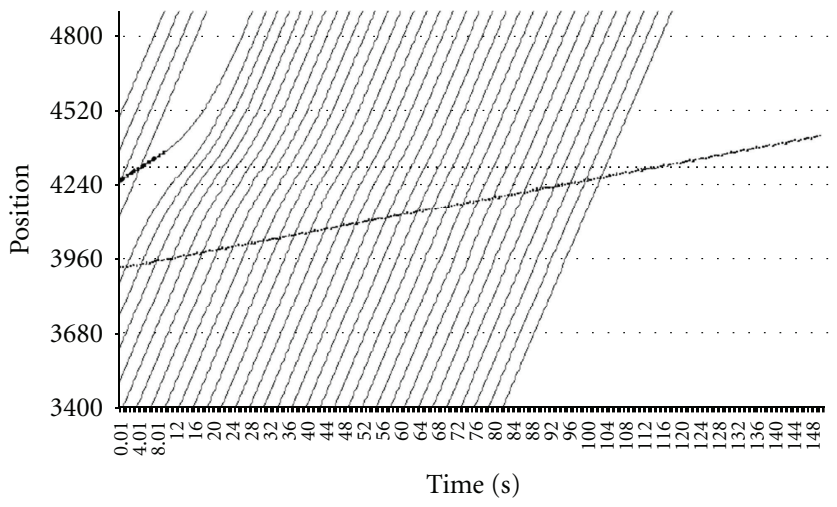

FIGURE 12: The positions occupied by cars in case of the lane entry algorithm, with $V_{\mathrm{NC}}\left(t_{0}\right)=11.15 \mathrm{~m} / \mathrm{s}$; distance between cars $250 \mathrm{~m}$. The thicker line represents $\operatorname{car}_{\mathrm{NC}}$ being on the lane next to the reserved lane; when it becomes a normal line, the car has entered the reserved lane.

The ILRSH's general architecture has been described, together with its components: reservation subsystem, monitoring subsystem, lane entry assistance component, and lane exit assistance component. We presented architectural design details for these components, together with algorithms to assist the driver take safe decisions, without disturbing 
the normal flow of cars on the highway. We also presented evaluation results that demonstrate the feasibility of the system.

The proposed system is capable to increase the efficiency of the highway. Without ILRSH cars are forced to travel at a lower speed (depending on the local traffic regulations). When the ILRSH system assists the driver, the result is a lane where cars travel at a higher speed, while still travelling in safe driving conditions. With the support of modern technology drivers are, therefore, assisted in taking the most efficient driving decisions. In turn, the capacity of the highway increases because the flow of cars increases per time interval.

Algorithms similar to speculative execution could be further implemented in the management of traveling cars subsystem, in order to better arrange the traveling cars and prepare the free slots for those cars that have just entered the highway and, since they are having a valid reservation, they are expected to request to enter the reserved lane as soon as they get on the lane near the reserved lane. (The speculative execution may start when BEnC_PoR monitoring boxes identify such cars). These new algorithms may require "positioned on road" monitoring boxes to send more information.

\section{Acknowledgments}

The research presented in this paper is supported by national project "TRANSYS-Models and Techniques for Traffic Optimizing in Urban Environments," Contract no. 4/28.07.2010 and Project CNCSIS-PN-II-RU-PD ID: 238. The work has been cofunded by the Sectoral Operational Programme Human Resources Development 2007-2013 of the Romanian Ministry of Labour, Family and Social Protection through the Financial Agreement POSDRU/89/1.5/S/62557.

\section{References}

[1] A. Duany, E. Plater-Zyberk, and J. Speck, Suburban Nation: The Rise of Sprawl and the Decline of the American Dream, North Point Press, 2000.

[2] J. Einbeck and J. Dwyer, "Using principal curves to analyse traffic patterns on freeways," Transportmetrica, vol. 7, no. 3, pp. 229-246, 2011.

[3] N. Ravi, S. Smaldone, L. Iftode, and M. Gerla, "Lane reservation for highways (position paper)," in Proceedings of the 10th International IEEE Conference on Intelligent Transportation Systems (ITSC '07), pp. 795-800, Seattle, Wash, USA, October 2007.

[4] A. J. Aman, D. C. Nuñez, M. Hamideddin, P. Laungani, W. Abbo, and G. Donohue, "Evaluation of congestion pricing for management highway in Seattle," in Proceedings of the IEEE Systems and Information Engineering Design Symposium (SIEDS '09), pp. 258-263, Charlottesville, Va, USA, April 2009.

[5] T. Hau, "Economic fundamentals of road pricing: a diagrammatic analysis, part I-fundamentals, and economic fundamentals of road pricing: a diagrammatic analysis, part II-relaxation of assumptions," Transportmetrica, vol. 1, no. 2, pp. 81-117, 2005.

[6] S. Mazilu, M. Teler, and C. Dobre, "Securing vehicular networks based on data-trust computation," in Proceedings of 13th
International Symposium on Symbolic and Numeric Algorithms for Scientific Computing (SYNASC '11), Timisoara, Romania, 2011. 

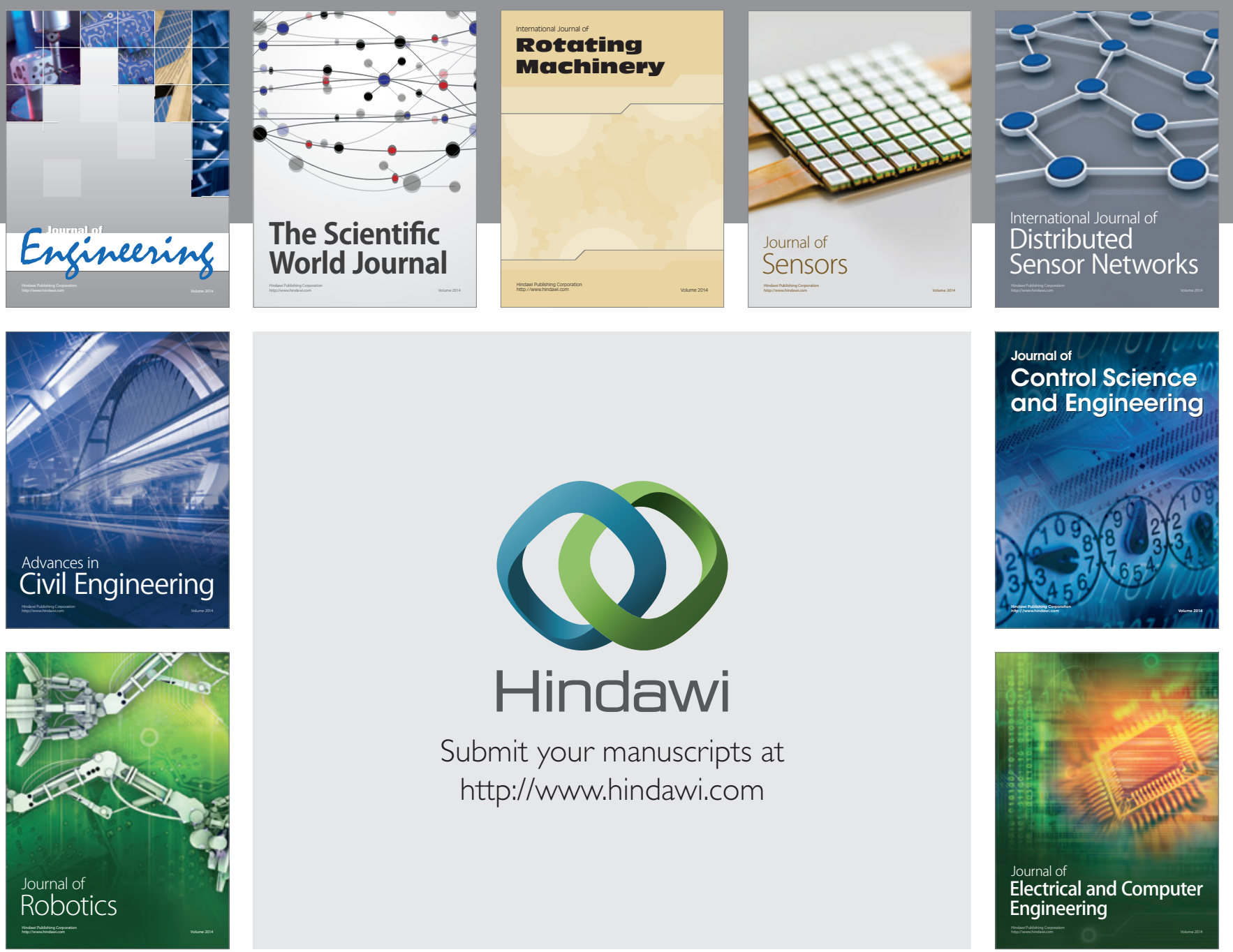

Submit your manuscripts at

http://www.hindawi.com
